\title{
25 Meter Rifle Shooting Training Kit
}

\author{
Mavillapalli Nikhitha a,1, Shobana D a, Anne Sylvia S a, Batta Hemasree a, \\ Priyadharshini $\mathrm{P}^{\mathrm{a}}$ \\ ${ }^{a}$ Department of Electrical and Electronics Engineering, Panimalar Institute of \\ Technology, Chennai, Tamilnadu,India
}

\begin{abstract}
The goal of this project is to obtain all of the details about the shotgun so that we can correct any mistakes made during practice. With so many undetectable errors made by operators at work, this device aids in locating and adjusting the timing of adjustments in accordance with their errors. This procedure is carried out by detecting deviations from the shooters while shooting from a distance. Following each session, all data is verified, and steps are taken to improve the shooters game and correct their mistakes. This device improves the quality of the archer while also assisting in the improvement of the game. It also appears to be a list of minor blunders that one may deal with. The arduino IDE, which does all mathematical and mathematical operations, is in charge of this device. The light attached to this device indicates the start and stop positions of the shooter, allowing the shooter to be trained to start and stop fire.
\end{abstract}

Keywords. Gun Shooting, Arduino, Android, Application, Bluetooth.

\section{Introduction}

The purpose of this project is to obtain all of the details about Rifle Shooter in order to correct any mistakes made during the process [1]. There are several unmistakable mistakes made by the shooter in operation, and this device aids us in detecting them and changing the timing of their mistakes. The process is improved even further by detecting deviations from the shooters while shooting from a distance. The coach and the player (shooter) analyze this deviation. After each session, all data is verified, and steps are taken in accordance with the errors that occurred. The device improves the quality of the archer and, as a result, aids in the improvement of the game. The project has a large arduino Nano controller, arduino Mega, which is utilized for all statistical and mental units in all calculations. The sensors employed in this project are bluetooth modules, which are similar to input devices. But the system is for viewing telemetry data. The sensors are connected to read various posters and user status to detect mistakes made by them and to correct the mistake made by them.

Mavillapalli Nikhitha, Department of EEE, Panimalar Institute of Technology, Chennai, TN, India. E-mail: mavillanikhitha723@gmail.com. 


\section{Existing System}

Smart Detector that will be able to detect reeds above the knee level and, more importantly, helps to navigate around large objects alone with the help of RFID (radio frequency detection system). RFID technology is precious, precise, and compatible with Braille blocks [2]. The detector has a microcontroller and an RFID reader on the bottom that can scan a piece of RFID cards on the floor and assist the user in navigation. This can only provide enough user vibration for error resolution. After shooting people, User Target Detector programmes are created, but only a handful can provide powerful interaction to adapt to changes. Outside, it doesn't work as well. The Target Detector employs a precise positioning system, wireless communication, a portable computer, and a voice communication interface to test people and assist them in making a mistake in both familiar and unfamiliar environments. It only provides a valuable input of materials that do not provide bodybuilding processes. This is a significant disadvantage of the target detector [3-5].

\section{Proposed System}

Our project contains the Arduino Mega controller is used for all arithmetic and logic units. The Bluetooth, RT, and SD ard modules were employed as input devices in this project. The NRF module, display, audio amplifier, and speaker make up the output system. The sensors are connected to read various posters and user status in order to detect and correct mistakes made by them. Using the display, the gun manager selects the shooting mode with the android system and the controller leads to the display output, the audio output, and changes the brightness depending on the functions selected by the users.

\section{Hardware Description}

\subsection{Arduino Nano}

Arduino is based on computer hardware and in electronic platform it is open sourced and this is very easy for using in environment. This is designed for persons interested in making objects, artists, hobbyists or collaborative spaces. It senses the environment in absorbing the input from other sources and it can affects the environment in controlling the engines, light and objects in it. The microcontroller board is configured by language of Arduino system and Arduino development environment. The projects based on Arduino can create on their own ideas or interacting with software in computer as like in processing, flash. This Nano type Arduino is powered by fixing a mini-B USB, 5V external power supply in the pin 27. Then the source for power will be automatically selected from the power source of highest value.

\subsection{Arduino Mega}

This mega type of Arduino micro controller board is ATMega2560 based model. This type of Arduino holds 54 digital input and output pins in which 15 of which used 
for PWM output, 16 analog input pins, 4 UART, 16 MHZ crystal oscillator, communication USB jack, reset button and ISCP header in it. This type of Arduino has all things which is needed in supporting the micro controller. This device can be used easily connected with the USB to the computer or it can be connected to power with an AC to DC adapter or can be stored with the help of battery. This Mega2560 Arduino has come to be an update to Arduino mega. This mega Arduino board is more consistent with more UNO shoes with previous boards Diecimila.

\subsection{Bluetooth}

HC-05 is a type of module which combines two-way multi functionality in our project. We can use this type of module to combine the two micro controllers to connect with any device also the functionality of Bluetooth with it such as laptop or mobile phones. Already many android applications are created to make this process much easier. This type of module will be able to communicate with USART at a rate of 9600 band and this makes the work easier to support USART with any type of micro controller. In this we can also make fixed values using the mode of command option. This module might be the good option when we look for a wireless module which transmit the data to a micro controller from computer or mobile phone. This module does not transfer multimedia application like images, songs and we can refer for that CSR8645 module. This HC-05 combines of two active modes namely, one in which data mode can be send and receive data from Bluetooth devices. Whereas, the other for AT command mode to change the default device settings. We can use this device by using the pin as mentioned in the description of the pin.

\subsection{Speaker}

Digital Speaker works on the 8002-speaker amplifier IC. This speaker is used as a buzzer or for Arduino with high playing quality. The volume of the speaker is managed with a potentiometer. This module has large benefits like intuitive interface, long life of working, compact text. This module can be combined with Arduino input and output expansion shield in related and sound creation.

\subsection{Power Supply}

As a result, as compared to other $12 \mathrm{~V}$ batteries, this one is relatively light. The battery life will stay longer upto $3-5 x$ than the SLA or VRLA battery equivalent. The light weight battery has $30 \%$ of SLA weight with $12 \mathrm{v}$ of system charging in it. It remains more strength through extraction when compared to lead. This can be end up in payment status left out being comprised. It is much safer than traditional lithium-ion batteries, sealed with a fabric. It is built on top of control and on top of release protection. The type of the product is of $12 \mathrm{v} 17 \mathrm{Ah}$ lead acid battery replacement. The various applications are ready to start with motor cycle, medical instruments, solar power, power supply in LED's, $12 \mathrm{v}$ DC power. This makes installation easy. It reduces costs and adds complexity. Battery life is more than 6 months for pre-configured transmitters and receivers. IrDA transceivers makes advantages in addition to reducing energy consumption. 


\section{Software Description}

\subsection{Arduino IDE}

Integrated Development with Arduino The environment consists of a text editor for writing code, a message area, a text console, a toolbar with bottom for common functions and a series of menus. It connects to the Arduino and genuine hardware to upload programs and communicate with them. Program written using Arduino software (IDE) are called sketches. These sketches are written in the text editor and saved with file extension. No. The editor has features for cutting/pasting and searching/replacing text. The message area given feedback while saving and exporting and display errors as shown in Figure 1.

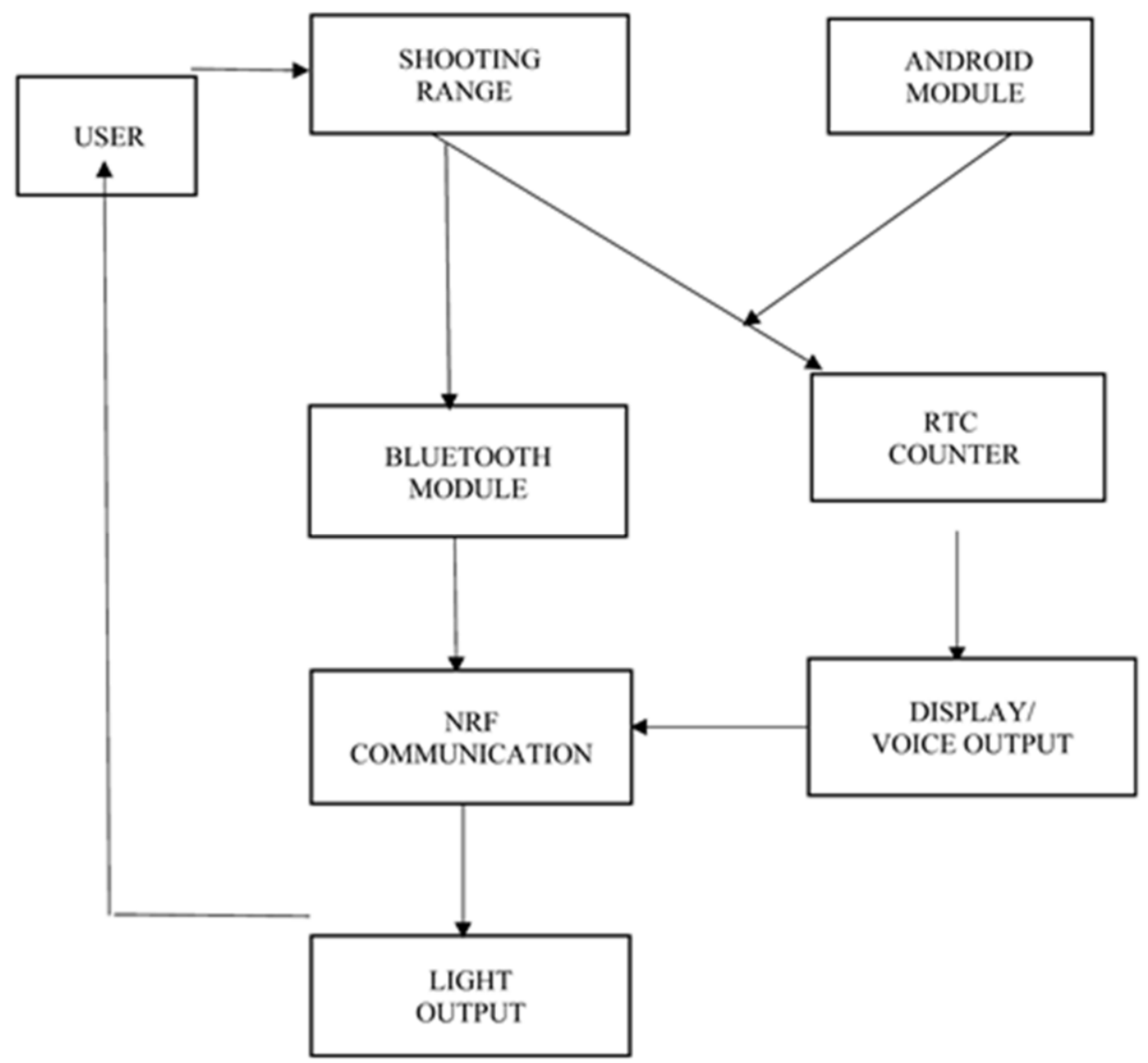

Figure 1. Block Diagram of Proposed System 


\subsection{Java}

The Java platform is an appropriate computer platform. All platforms are supported, from servers to mobile phones to smart cards. These java technology combines the business activity to produce a seamless, protective platform and network in our business. This platform of java has benefits from a wide community of active workers to develop and support to deliver products and services designed for java technology and to create the platform by an open standard organization and based on community known as the java community process. We can see java technology in laptops, internet, mobile phones and trackside at formula of one grand prix races.

\section{Hardware Results}

The output system consists of an NRF module, display, audio amplifier, and speaker. The sensors are connected to read various posters as well as user positions in order to detect errors made by them and correct the errors made by them. The gunman decides how to shoot with android application through display. And the controller leads to display output, audio output, and light switching based on user-selected functions as shown in Figure 2 and Figure 3.

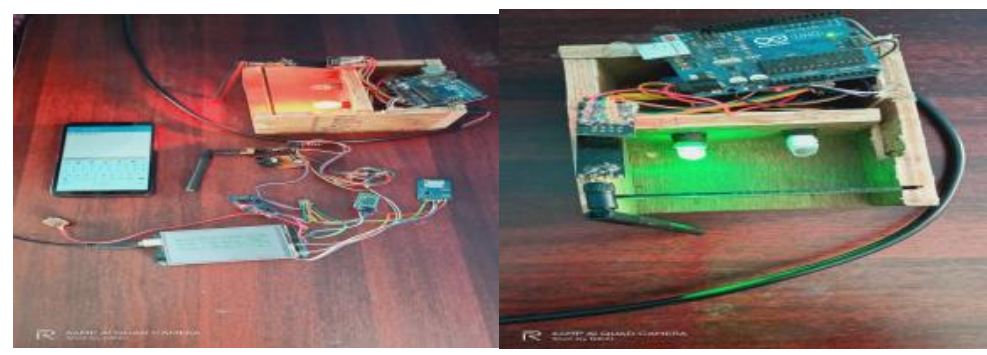

Figure 2. Sensor Display Controller

Figure 3. NRF Module

\section{Future Scope}

The system is utilised for a variety of purposes, including directing people in wheel chairs who are using IOT-based robotic operating systems. As IoT technology grows to be employed in almost all sectors, the associated production costs decrease. The idea of setting up this system is useful fry all games is possible legally. As the usage of IoT in the commercial sector continues to rise, production costs will be significantly reduced due to increased production, allowing IoT to be accepted in all games and into machine learning and archiving of sports and spontaneous training time.

\section{Conclusion}

This project includes a large Arduino Mega controller that is used for all arithmetic and logic units in all calculations. The Bluetooth, RT, and SD ard modules were employed as input devices in this project. The NRF module, display, audio amplifier, 
and speaker make up the output system. The sensors are connected to read a variety of posters and user statuses. Using the display, the gun manager selects the shooting mode with the android system. And the controller connects to the display output, the audio output, and adjusts the brightness based on the functions selected by the users. The proposed method reduces reliance on the central database, which may necessitate certain more time-consuming tasks in order to compile more data. Because each marker is managed separately to generate time, this program is incredibly useful and takes very little time.

\section{References}

[1] Exposito, I., \& Cuinas, I. (2013). Exploring the Limitations on RFID Technology in Traceability Systems at Beverage Factories. International Journal of Antennas and Propagation, 2013, 1-9. https://doi.org/10.1155/2013/916526

[2] Huang, Y., Cai, X., Kan, W., Qiu, S., Guo, X., Liu, C., \& Liu, P. (2017). A flexible dual-mode proximity sensor based on cooperative sensing for robot skin applications. Review of Scientific Instruments, 88(8), 085005. https://doi.org/10.1063/1.4998995

[3] S., T., \& S. Liu, J. W. (2013). Design and Implementation of RFID-Based Object Locators. In Radio Frequency Identification from System to Applications. InTech. https://doi.org/10.5772/53576

[4] Real, \& Araujo. (2019). Navigation Systems for the Blind and Visually Impaired: Past Work, Challenges, and Open Problems. Sensors, 19(15), 3404. https://doi.org/10.3390/s19153404

[5] Bai, J., Lian, S., Liu, Z., Wang, K., \& Liu, D. (2018). Virtual-Blind-Road Following-Based Wearable Navigation Device for Blind People. IEEE Transactions on Consumer Electronics, 64(1), 136-143. https://doi.org/10.1109/TCE.2018.2812498 